\title{
Modelo para Evaluar los Factores que Inciden en el Clima Laboral del GAD Provincial de Chimborazo
}

\section{Model to Evaluate the Factors that Influence the Labor Climate of the Provincial GAD of Chimborazo}

\author{
Ing. Rud Noemí Cunín Chimborazo ${ }^{1}$ \\ rncunin@pucesd.edu.ec \\ https://orcid.org/0000-0001-6124-509 \\ Dra. Olga Bravo Acosta ${ }^{2}$ \\ obravo@uteg.edu.ec \\ https://orcid.org/0000-0003-0558-3228
}

Recibido: 1/9/2020, Aceptado: 1/11/2020

\begin{abstract}
RESUMEN
La modernización de las entidades públicas y la creciente necesidad de mantener un clima laboral agradable es una temática importante en la actualidad, porque atribuye impacto directo en la percepción que el colaborador tiene con respecto a las prácticas administrativas, procesos, tecnología y otros factores, repercutiendo en su desempeño y por ende en el resultado de las organizaciones. Este trabajo tiene como propósito aplicar un modelo de evaluación que determine los factores que inciden en el clima laboral del GAD Provincial de Chimborazo, utilizando técnicas de recopilación de información, tales como entrevistas, observación y encuestas aplicadas a una muestra de 172 trabajadores. La investigación tiene un enfoque cuantitativo, diseño no experimental transversal y un alcance descriptivo - correlacional. Los resultados, al aplicar una combinación del Modelo de Litwin y Stringer (1968) \& Williams (2013), reflejaron que el clima laboral del GADPCH fue del 44\%. De acuerdo a cada dimensión, los siguientes: Estructura (67\%), Conflicto (41\%), Identidad (53\%), Cooperación / Apoyo $(42 \%)$, Recompensas $(29 \%)$ y Comunicación $(34 \%)$, resultando en un clima laboral regular. Es evidente la existencia de un cierto nivel de insatisfacción de los servidores públicos con respecto a su entorno laboral. Se presenta una propuesta, que contiene el planteamiento de estrategias de solución aplicadas a las dimensiones que obtuvieron la valoración más baja, mismas que están enfocadas a elevar la motivación, implementar nuevas técnicas de comunicación internas, gestionar arreglos satisfactorios por causa de un conflicto y también para fomentar el espíritu de trabajo en equipo.
\end{abstract}

Palabras clave: Clima laboral, Modelos de evaluación, Factores, Desempeño.

1 Maestría, Universidad Tecnológica Empresarial de Guayaquil, Ecuador.

2 Universidad Tecnológica Empresarial de Guayaquil, Ecuador. 


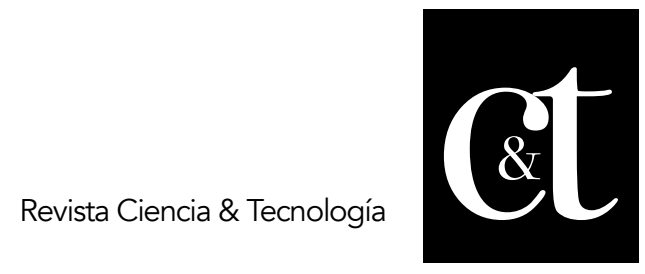

No. 29, 31 de enero de 2021

ISSN impreso: 1390 - 6321

ISSN online: 2661 - 6734

\begin{abstract}
The modernization of public entities and the growing need to maintain a pleasant work environment is becoming an important topic presently, because of its direct impact on the perception that the collaborator has regarding administrative practices, processes, technology and other factors, having an impact on their performance and therefore 0 the results of the organizations. This article aims at applying an evaluation model to determine those factors affecting the working environment in Provincial Government of Chimborazo, utilizing information gathering techniques, such as interviews, observation and surveys applied to a sample of 172 workers. The research involves a quantitative approach, transversal non - experimental design and with a descriptive correlational scope. Results, when applying a combination of the Litwin and Stringer (1968) \& Williams (2013) Models, reflected that the general working climate of the GADPCH was $44 \%$; also, each dimension shows: Structure $(67 \%)$, Conflict $(41 \%)$, Identity (53\%), Cooperation / Support (42\%), Rewards (29\%) and Communication (34\%); these, denoting a regular working environment is regular. It is evidently, the existence of a certain level of dissatisfaction among public employees. Finally, a proposal is presented, which includes strategies to be applied to those dimensions that obtained the lowest assessments, which are focused on raising motivation, implementing new internal communication techniques, managing satisfactory arrangements due to conflicts and also to encourage teamwork spirit.
\end{abstract}

Keywords: Work climate, evaluation models, factors, performance.

\title{
Introducción
}

Actualmente las organizaciones se vuelven cada vez más competitivas debido al mundo globalizado al que se enfrentan, no obstante la mayoría de empresas y especialmente las públicas han concentrado sus esfuerzos en el rendimiento y cumplimiento de objetivos, dejando como aspecto secundario la implementación de estrategias de mejoramiento y la creación de un ambiente de trabajo agradable para el cliente interno, pues en gran parte son los que establecen el éxito o fracaso de cualquier organización (Williams, 2013).

Si bien es cierto, (Rodríguez, 2015) afirma que las instituciones públicas se han convertido en el centro de cuestionamiento respecto al servicio de atención deficiente que actualmente brindan a sus clientes, y también están siendo objeto de una intensiva reflexión con respecto al desempeño, rendimiento y su calidad para gestionar la organización; para ello se requiere que los altos directivos centren su atención en mantener un personal satisfecho y adaptarlos hacia un ambiente laboral adecuado, pues según (Montoya \& Boyero , 2015), hoy en día el talento humano es considerado como un factor clave y principal fuente de ventaja sostenible para el éxito empresarial.

La presente crisis económica y social que está atravesando el país, añade una preocupación en el ámbito de las Administraciones públicas, porque la propia institución del empleo público está siendo denostada socialmente y sometida a todo tipo de tensiones políticas (Pecino, Mañas, Díaz, López, \& Llopis, 2015). Generando de cierta forma inestabilidad laboral, preferencias laborales, conflictos personales y problemas de salud ocasionados por el estrés principalmente en los trabajadores que no poseen nombramiento.

Por lo tanto, el GAD Provincial de Chimborazo a través de su apoyo para la realización de esta investigación, ha demostrado su preocupación, pues sus directivos afirman la 


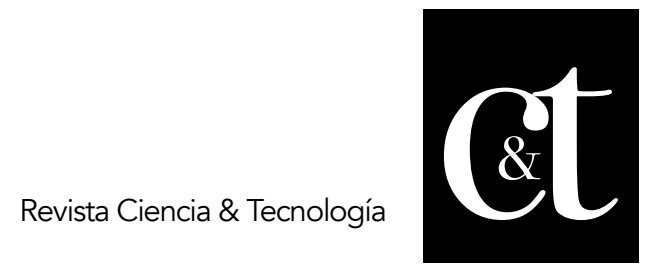

No. 29, 31 de enero de 2021

ISSN impreso: 1390 - 6321

ISSN online: 2661 - 6734

existencia de múltiples problemas internos: (Inseguridad laboral, estrés, desmotivación, falta de compañerismo, entre otros), lo cual repercute de manera directa en un bajo desempeño de los servidores públicos (78\%). En este sentido, el propósito del presente artículo radica en la aplicación de un modelo de evaluación que determine los factores que inciden en el clima laboral del Gobierno Autónomo Descentralizado de la Provincia de Chimborazo; puesto que de acuerdo a manifestaciones realizadas por el Jefe de Talento Humano del GADPCH hasta la actualidad no se ha realizado un estudio relacionado al clima laboral en dicha entidad pública, lo cual ha generado retrasos en los procesos, insatisfacción laboral, en fin múltiples problemas y transformaciones negativas a nivel organizacional.

Ahora bien, para mayor sustento de la investigación y como guía para la realización del presente estudio, se procedió a revisar investigaciones que han abordado la misma situación, iniciando por el estudio de una tesis de maestría desarrollada por (Williams, 2013), con el tema "Estudio Diagnóstico de Clima Laboral en una Dependencia Pública", cuyo propósito consistió en conocer la tendencia general de la percepción del talento humano sobre el clima laboral dentro de una dependencia municipal, misma que se enfocó en 5 dimensiones relacionadas a procesos de comportamiento organizacional y a las necesidades de la entidad: liderazgo, comunicación, motivación, espacio físico y trabajo en equipo. En relación a las dimensiones evaluadas los servidores públicos reflejaron un alto nivel de insatisfacción hacia el ambiente donde se desempeñan, puesto que los resultados estadísticos están por debajo de la media con un $46 \%$ respectivamente.

Asimismo el estudio de (Jiménez \& Mosquera, 2017), cuyo objetivo radica en analizar la incidencia del clima organizacional en el desempeño laboral de los colaboradores de un departamento financiero en una entidad pública, con énfasis en las condiciones físicas de trabajo, motivación, liderazgo, equidad, trabajo en equipo, responsabilidad y comunicación. Como resultado del proceso investigativo se estableció que el ambiente laboral se encuentra en una situación de riesgo con un $43 \%$ para lo cual proponen un plan de mejora que permita mejorar su entorno laboral.

Es importante recalcar que los dos trabajos investigativos antes mencionados llegaron a la conclusión de que la desmotivación y la falta de trabajo en equipo son los principales factores que ocasionan un cuello de botella y que afectan radicalmente al normal funcionamiento de las instituciones, también, acuerdan que una adecuada evaluación del clima laboral es la clave para el éxito institucional que condiciona el comportamiento y actitudes de los colaboradores.

Por su parte, la tesis magistral de (Rodríguez, 2015), denota un clima laboral favorable y aspectos de la cultura organizacional alentadores, donde la satisfacción de los funcionarios públicos se revela optimista y percibido de manera positiva, sin embargo al tratarse de una institución pública, cuenta con una estructura burocrática, es decir las normas, reglamentos, trámites y procedimiento no facilitan un trabajo fluido. De igual manera se puede evidenciar resultados positivos y un nivel de percepción satisfactorio por parte de los colaboradores con respecto al clima laboral, en el estudio realizado por (Salvatierra, 2016), no obstante debe mejorar el sistema de recompensas para mejorar su motivación y por ende su desempeño. 


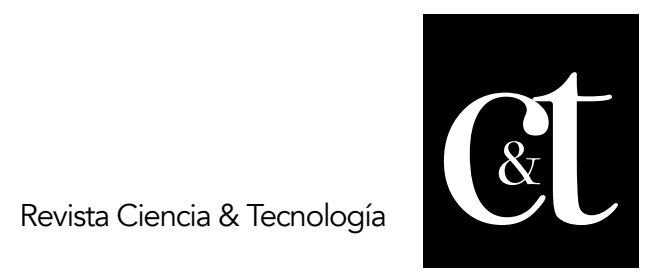

No. 29, 31 de enero de 2021

ISSN impreso: 1390 - 6321

ISSN online: 2661 - 6734

\section{Metodología}

Posterior a una serie de reflexiones, teorías, modelos y revisiones teóricas manifestadas por distintos autores a través del tiempo se trata de sustentar el instrumento de estudio científico, tomando en cuenta el objetivo y la formulación del problema de la investigación en cuestión. Entonces, es conveniente destacar el modelo que permitió medir los factores que inciden en el clima laboral del GADPCH, mismo que se desarrolló a través de la combinación del Modelo de Litwin y Stringer (1968) y el Modelo de Williams (2013). En este sentido se escogió el Modelo de Litwin y Stringer (1968), porque según los estudios expuestos en el marco teórico es el modelo más utilizado por los investigadores para evaluar el clima laboral por su fiabilidad y precisión, además porque es adaptable a cualquier tipo de organización y porque se enfoca en la estructura organizacional, percepción y comportamiento de los individuos referente a su entorno laboral, rescatando las dimensiones de (estructura, conflictos, identidad, cooperación / apoyo, recompensas). Del mismo modo, se eligió la aportación del reconocido Modelo de Williams (2013), por ser un modelo de medición de clima laboral adaptado especialmente para entidades públicas; y porque a través de la dimensión de (comunicación) se puede medir una de las problemáticas existentes en el GADPCH. Cabe indicar que los indicadores se adaptaron de acuerdo a las necesidades y requerimientos existentes en la entidad. A continuación en la Tabla 1 se presenta la escala de Likert de cinco puntos para valorar los distintos indicadores con mayor precisión, propuestos en el estudio.

\section{Resultados}

Tabla 1: Rango de calificación para el modelo propuesto

\begin{tabular}{cl|cc}
\hline Esca & Criterio & Rango & \\
\hline 1 & Totalmente en desacuerdo & $76 \%$ al & Clima óptimo \\
2 & En desacuerdo & $51 \%$ al $75 \%$ & $\begin{array}{c}\text { Clima } \\
\text { aceptable }\end{array}$ \\
3 & Ni en acuerdo, Ni en desacuerdo & $26 \%$ al $50 \%$ & $\begin{array}{c}\text { Clima regular } \\
\text { Clima } \\
4\end{array}$ \\
& De acuerdo & $1 \%$ al $25 \%$ & deficiente \\
\hline
\end{tabular}

Elaboración propia

La investigación es exploratoria, por ser una temática que no ha sido estudiada en el GADPCH y además porque se utiliza en la etapa inicial del estudio a fin de que proporcione un acercamiento superficial al problema, para posteriormente indagar una problemática más puntual y rigurosa sobre el talento humano de la entidad en cuestión. Es de tipo descriptivo debido al proceso de detallar la situación existente del fenómeno observado en cada una de las variables sujetas a estudio y plasmar una fotografía de la situación actual por medio del análisis particular y la descripción de cada una de ellas a fin de medir las distintas dimensiones proporcionadas por el modelo de evaluación de clima laboral a aplicarse. Tiene un enfoque cuantitativo porque la recopilación de información se basa en la obtención de datos reales y objetivos más no en supuestos. También, se hizo uso del método lógico cuando se redactó el marco teórico y para comparar entre los distintos modelos el más adecuado para evaluar el 


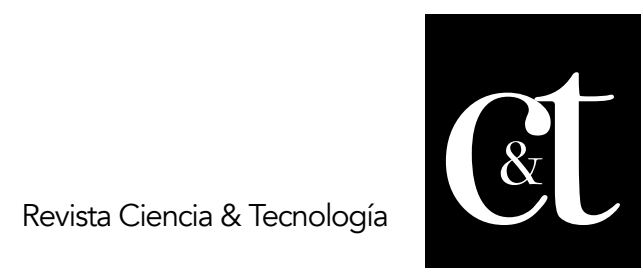

No. 29, 31 de enero de 2021

ISSN impreso: 1390 - 6321

ISSN online: 2661 - 6734

clima laboral del GADPCH. Asimismo el método empírico fue necesario al momento de realizar la investigación de campo y la recolección de información.

\section{Resultados y discusión}

Resulta oportuno destacar que, este capítulo permite reflejar los resultados obtenidos en la investigación de campo efectuada a los 172 servidores públicos del GADPCH, referente al clima laboral; al mismo tiempo interpretar, discutir y comparar dichos resultados, en primer lugar en función del clima laboral en su totalidad, después de acuerdo a las seis dimensiones propuestas por el modelo y por último considerando cada uno de los ítems de las dimensiones: (estructura, conflicto, recompensas, comunicación, identidad y cooperación/apoyo).

\section{Clima laboral a nivel general}

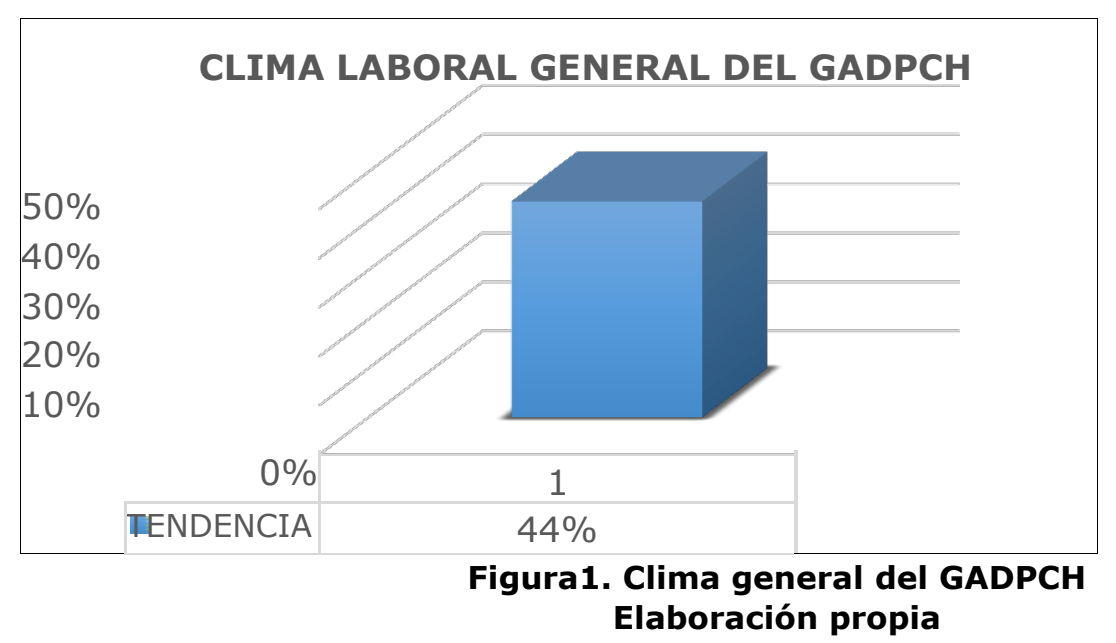

Mediante los resultados obtenidos de las encuestas aplicadas a los empleados, se determinó que el clima laboral del GADPCH en general corresponde al $44 \%$, en relación a las dimensiones estudiadas de estructura, conflicto, recompensas, comunicación, identidad y cooperación/apoyo; lo cual denota que el clima laboral cualitativamente es regular, dicho de otro modo, es evidente la existencia de un cierto nivel de insatisfacción de los colaboradores con respecto a su entorno laboral, siendo urgente la implementación de estrategias de mejoramiento. 


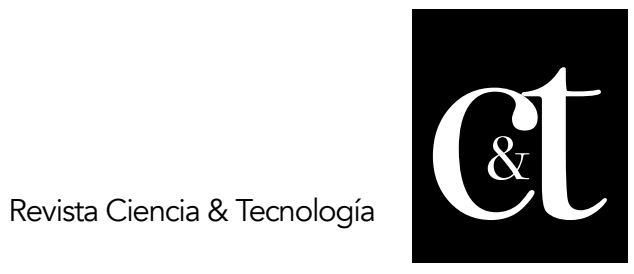

No. 29, 31 de enero de 2021

ISSN impreso: 1390 - 6321

ISSN online: 2661 - 6734

\begin{tabular}{|c|c|c|}
\hline DIMENSIONES & PROMEDIO & $\begin{array}{l}\text { RANGO DE } \\
\text { VALORACIÓN }\end{array}$ \\
\hline Estructura & $67 \%$ & Clima Aceptable \\
\hline Conflicto & $41 \%$ & Clima Regular \\
\hline Identidad & $53 \%$ & Clima Aceptable \\
\hline Cooperación / apoyo & $42 \%$ & Clima Regular \\
\hline Recompensas & $29 \%$ & Clima Regular \\
\hline Comunicación & $34 \%$ & Clima Regular \\
\hline
\end{tabular}

\section{Elaboración propia}

En base a los datos obtenidos, se establece que las dimensiones que obtuvieron la mayor calificación fueron "Estructura" e "Identidad", dado que los servidores públicos aducen que las políticas, reglamentos y procedimientos son claros dentro de la entidad y también porque disponen de recursos necesarios para cumplir con sus obligaciones. En este sentido dichos resultado se ven reflejados en el sentido de pertenencia, en otras palabras, los colaboradores se sienten orgullosos de formar parte del GADPCH. Por el contrario, las dimensiones de "Conflicto", "Cooperación/Apoyo", "Recompensas" y "Comunicación" fueron las menos calificadas, porque los encuestados perciben que no se les otorga reconocimiento especial por su buen desempeño, también por la falta de apoyo para estimular su crecimiento profesional y además porque en la entidad no se trabaja por mantener una comunicación que promueva la integración, unión y compañerismo dentro los distintos grupos de trabajo.

\section{Clima laboral según dimensiones del modelo propuesto y sus ítems}

\section{Estructura}

Se determinó que el ítem correspondiente a las políticas, reglamentos y procedimientos son claros dentro de la institución, alcanzó la calificación más alta $(84 \%)$, seguido del ítem ubicado en segundo lugar en importancia correspondiente a la formalidad para dar paso a un trámite no incide en los resultados ( $83 \%$ ) y además que los niveles jerárquicos no afectan al proceso de toma de decisiones con un (55\%), posicionándolos según la escala de valoración en un nivel óptimo de clima laboral, sin embargo estos indicadores comienzan a verse afectados debido a que las actividades no tienen congruencia con el puesto de trabajo, que de cierto modo están formando un cuello de botella para el normal funcionamiento de las actividades. 


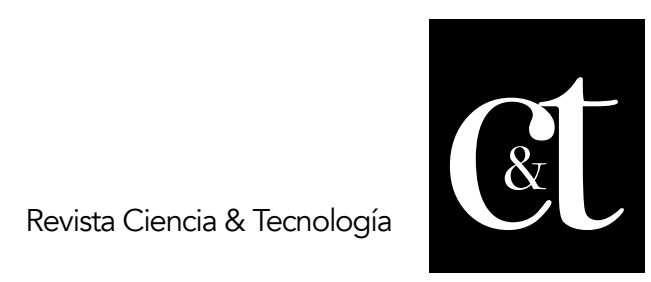

No. 29, 31 de enero de 2021

ISSN impreso: 1390 - 6321

ISSN online: 2661 - 6734

\section{Identidad}

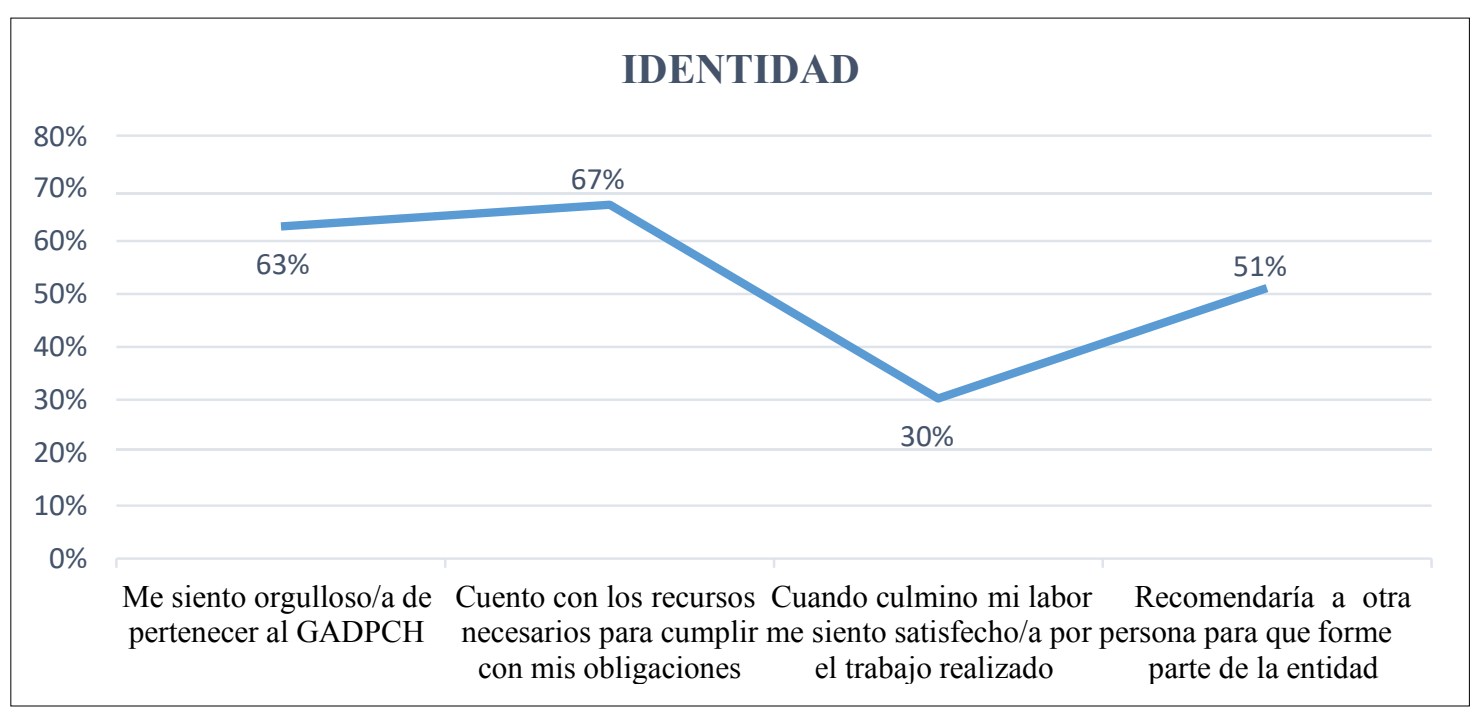

Figura 3: Dimensión de Identidad.

Elaboración propia

En lo que se refiere a la percepción que el personal del GADPCH tiene con respecto a la identidad, se puede mencionar que esta dimensión acumuló un promedio del 53\%, demostrando un clima laboral aceptable. Ahora bien al comparar los distintos indicadores, se identifica claramente que la entidad dispone de recursos y herramientas necesarias para que sus colaboradores puedan cumplir con sus labores diarias; consecuentemente se señala que el $63 \%$ de los encuestados dicen sentirse orgullosos de pertenecer al GADPCH; asimismo un porcentaje mayoritario ( $51 \%$ ), expresaron que estarían dispuestos a recomendar a otra persona para que forme parte de la entidad; ubicándolos también según el rango de valorización en un nivel aceptable. El último ítem, satisfacción por el trabajo realizado obtuvo el puntaje más bajo, obteniendo de esta manera un clima laboral regular; denotando necesidad de aplicar medidas; en este caso una estrategia serían actividades acordes a su puesto de trabajo, en función de sus capacidades y conocimientos. 


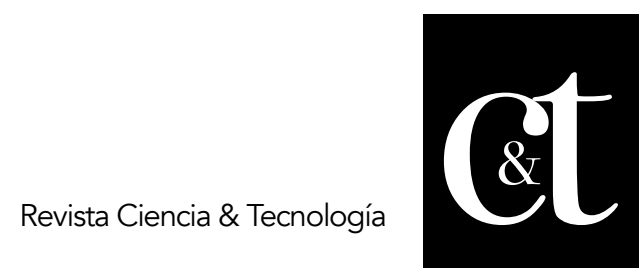

No. 29, 31 de enero de 2021

ISSN impreso: 1390 - 6321

ISSN online: 2661 - 6734

\section{CONFLICTO}

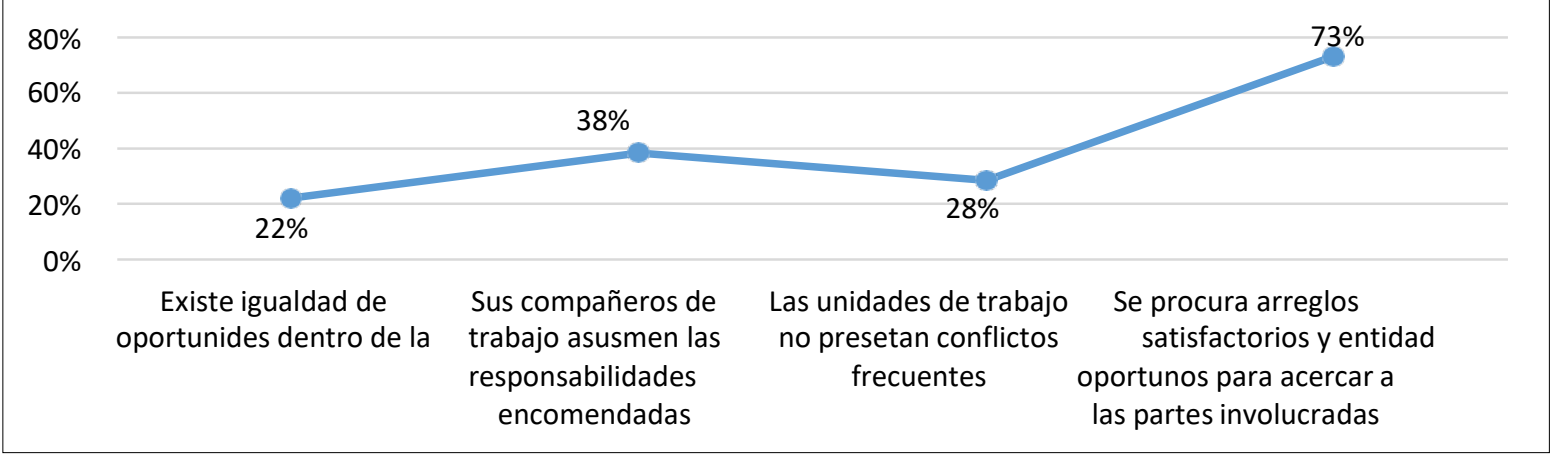

\section{Figura 4: Dimensión de Conflicto Elaboración propia}

En cuanto a la percepción que el talento humano tiene con respecto al conflicto, se revela que alcanzó un promedio del $41 \%$, representando clima regular según las calificaciones establecidas por el modelo aplicado; siendo el ítem con mayor representatividad $(73 \%)$, la gestión de los superiores para lograr arreglos satisfactorios y oportunos a fin de lograr un nivel aceptable. Es preciso notar que de acuerdo a la información recopilada, la desigualdad de oportunidades, la falta de responsabilidad para asumir tareas entre sus compañeros, están provocando que las distintas unidades de trabajo presenten conflictos frecuentes y un clima laboral regular, como se puede observar en los resultados obtenidos de los tres indicadores de dicha dimensión.

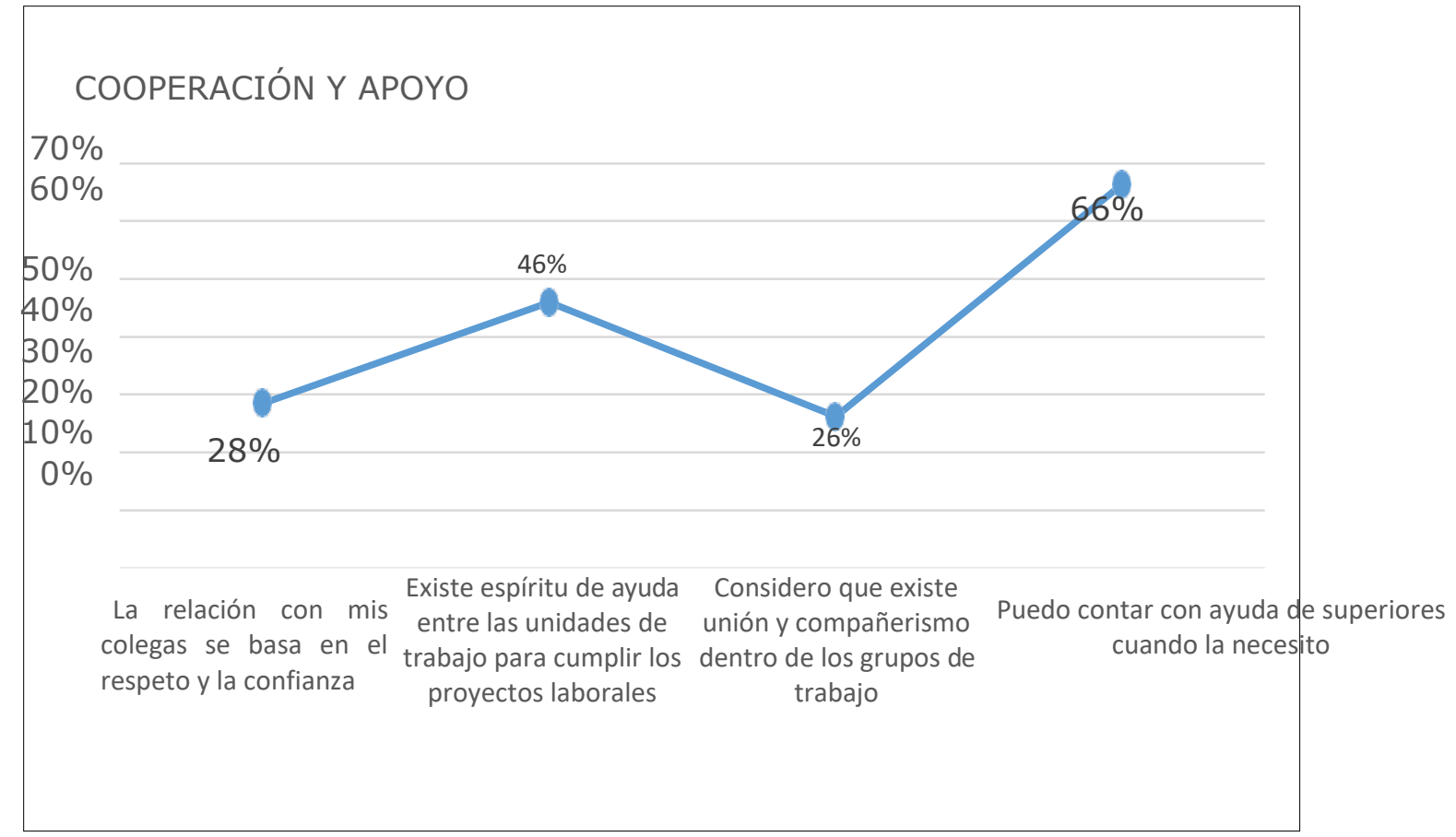

Cunín, Bravo. Modelo para evaluar los factores que inciden en el clima laboral del GAD Provincial de Chimborazo 


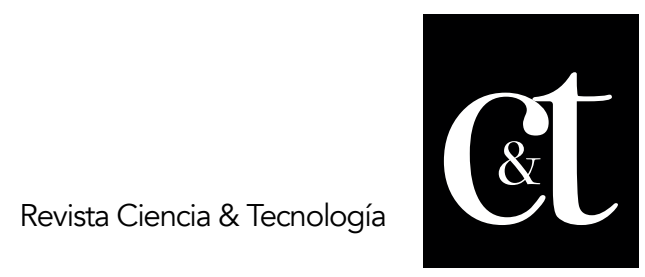

No. 29, 31 de enero de 2021

ISSN impreso: 1390 - 6321

ISSN online: 2661 - 6734

La cooperación y apoyo es la tercera dimensión mayor valorada en la presente investigación con un promedio del $42 \%$, no obstante pese al porcentaje alcanzado, esto denota un clima laboral regular. Por su parte los ítems referentes al compañerismo y la confianza entre los grupos se encuentran ubicados en los niveles más bajos con un $26 \%$ y un $28 \%$ respetivamente, seguido del indicador con relación al espíritu de ayuda entre los grupos para cumplir con los proyectos laborales con $46 \%$, ubicándolos en un nivel regular. Sin duda alguna se puede identificar que en un grado aceptable se encuentra el indicador relacionado al espíritu de ayuda que los superiores tienen hacia su personal. En fin, considerando todo lo anteriormente expuesto, los resultados denotan la necesidad de trabajar en actividades y estrategias que fomenten el compañerismo y sobre todo impulsar la confianza entre los grupos, para lograr un ambiente laboral más atractivo.

\section{Recompensas}

Al analizar los cuatro ítems relacionados a la dimensión de recompensa, se puede establecer que a nivel general esta dimensión obtuvo el promedio más bajo (29\%) de todas las dimensiones estudiadas, adquiriendo según el cuadro de valoración propuesta por el modelo un clima regular. Asimismo, dentro de este análisis en la figura 6 se observa que el ítem correspondiente a si la entidad proporciona seguridad y estabilidad laboral, alcanzó la mayor valoración es decir un nivel aceptable; sin embargo es importante hacer notar que esta información se corrobora con los registros obtenidos del GADPCH, dónde 162 servidores públicos cuentan con nombramiento, mientras que los

152 restantes disponen de contratos ocasionales, siendo esta una razón potencial para una baja motivación, puesto que muchos temen perder el único medio de sustento para sus familias. Además, según entrevista mantenida con el Director de Talento Humano del GADPCH, la última vez que se otorgaron partidas por nombramiento fue en el año 2012 y que recién es este mes de octubre del 2018 se otorgarán 10 partidas como una oportunidad que debe ser aprovechado tanto por trabajadores internos como externos.

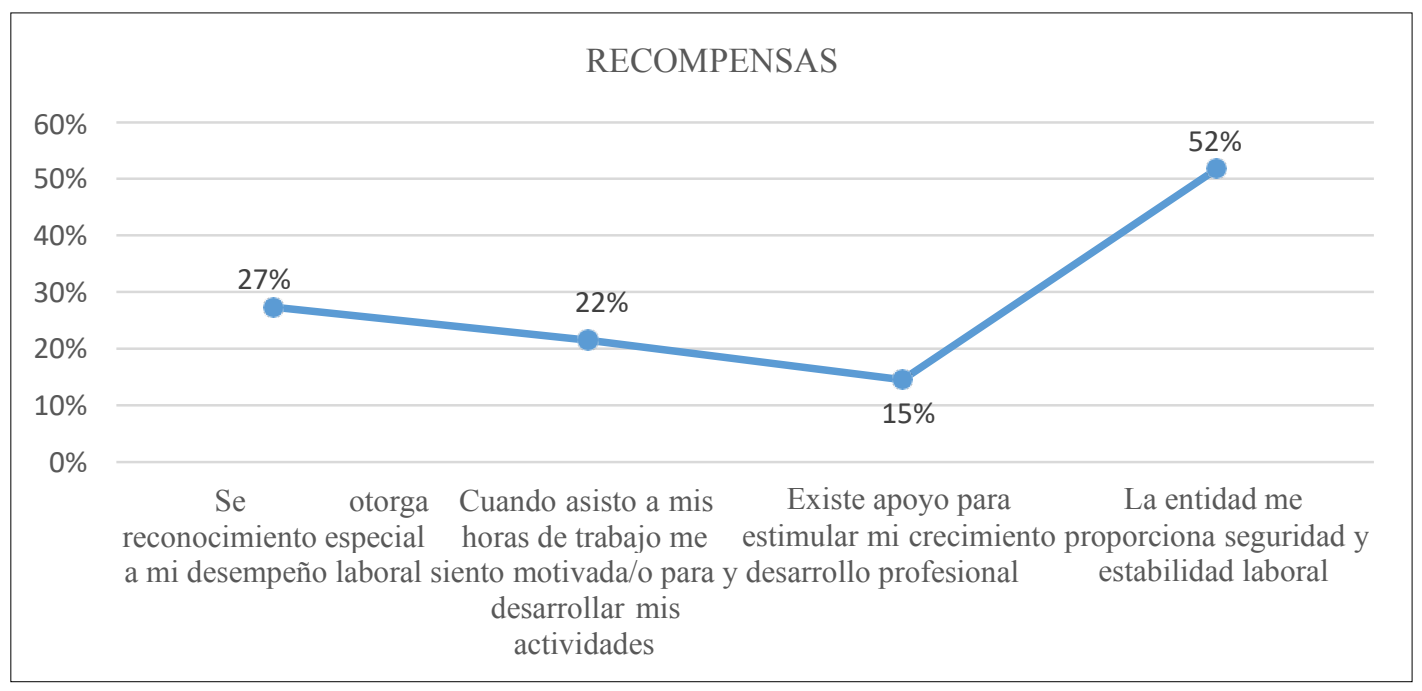

Figura 6: Dimensión de Recompensas. Elaboración propia

Revista Científica Ciencia y Tecnología Vol 21 No 29 págs. 72-83

http://cienciaytecnologia.uteg.edu.ec 


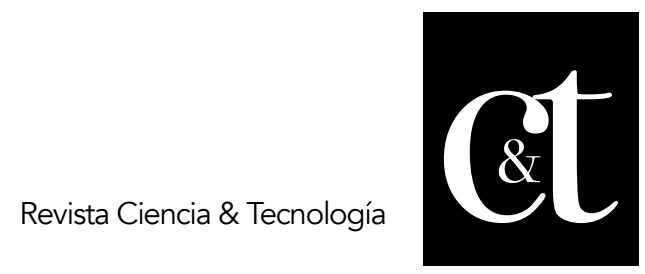

No. 29, 31 de enero de 2021

ISSN impreso: 1390 - 6321

ISSN online: 2661 - 6734

Del mismo modo, se puede notar que los ítems referentes a si se otorgan reconocimientos especiales por el buen desempeño laboral, cuando asiste a las horas de trabajo se sienten motivados para desarrollar sus actividades diarias y por último si existe apoyo para estimular el crecimiento y desarrollo profesional, fueron los que recibieron la más baja calificación equivalente a un nivel regular, con un $27 \%, 22 \%$ y $15 \%$ respectivamente. Visto desde otro punto, el escaso presupuesto para capacitaciones, la falta de estímulos, reconocimiento y la inestabilidad laboral en trabajadores con contrato ocasional, están provocando a que la entidad cuente con un personal desmotivado, lo cual conlleva al incumplimiento de los objetivos propuestos.

\section{Comunicación}

Se determinó la percepción del Talento Humano del GAD Provincial de Chimborazo, en torno a la comunicación, los datos señalan que fue la segunda dimensión menos valorada con el $34 \%$ de representatividad, situándolo en un nivel de clima laboral regular. Entonces sobre los ítems de esta dimensión se comprobó que los canales de comunicación internos no se encuentran claramente definidos, igualmente la información que es proporcionada por los superiores es deficiente es decir no es fluida, cabe agregar también el descontento de parte de los servidores públicos por la falta de gestión para mantener una comunicación que promueva la integración y cohesión de los equipos, pues en este caso los tres ítems acumularon un porcentaje sumamente bajo $(23 \%, 27 \%$ y $27 \%)$ respectivamente, lo cual se asemeja con un nivel regular. Después de las consideraciones anteriores, se identificó que en muchos casos la información no llega a todo el personal, por lo que generalmente se cometen fallas y retrasos en la presentación de los proyectos. Como medidas correctivas es preciso hacer uso de las TICs especialmente de medios gratuitos como son las herramientas tecnológicas e internet, con el propósito de enviar comunicados y que a su vez sea capaz de cubrir a todos y cada uno de los miembros que laboran dentro de la entidad.

\section{Conclusiones}

La investigación permitió conocer, por medio de la revisión teórica los modelos más reconocidos para evaluar el clima laboral en distintos tipos de organizaciones, utilizando dicha información se adaptó un modelo a través de la combinación del Modelo de Litwin y Stringer (1968) \& Williams (2013) para medir el clima laboral actual del GADPCH. Se optó por el Modelo de Litwin y Stringer (1968) por su fiabilidad y precisión, además porque principalmente se enfoca en la estructura organizacional, percepción y comportamiento de los individuos referente a su entorno laboral, rescatando las dimensiones de (estructura, conflictos, identidad, cooperación / apoyo, recompensas). Asimismo, se eligió el Modelo de Williams (2013), porque la dimensión de (comunicación) ayudó a medir una de las problemáticas existentes en la institución.

Se determinó que las encuestas aplicadas a la muestra seleccionada utilizando el modelo propuesto, permitió medir los factores que inciden en el clima laboral del GADPCH. En este sentido, las dimensiones que obtuvieron la mayor calificación fueron Estructura (67\%) e Identidad (53\%) categorizándose en un nivel aceptable de clima laboral, no obstante la dimensión de recompensas, obtuvo la calificación más baja correspondiente al $29 \%$, seguido de la dimensión de comunicación con un $34 \%$, asimismo las dimensiones de conflicto y cooperación/apoyo con un $41 \%$ y $42 \%$ respectivamente, posicionándose en un nivel regular. Cabe agregar, que pese a que la 


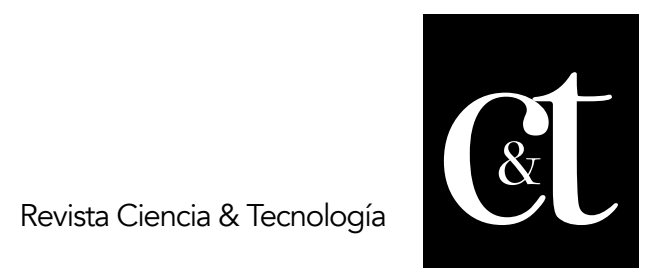

No. 29, 31 de enero de 2021

ISSN impreso: 1390 - 6321

ISSN online: 2661 - 6734

estructura organizacional es buena y los colaboradores se sienten identificados como parte importante de la entidad, el clima laboral a nivel general del GADPCH (41\%) no es adecuado dada las calificaciones obtenidas en las otras dimensiones que han sido evaluadas. Al realizar una comparación con los estudios realizado por Jiménez \& Mosquera (2017) y Williams (2013) en entidades públicas, los resultados no variaron significativamente puesto que obtuvieron un porcentaje similar equivalente al $43 \%$ y $46 \%$ ubicándolos en un clima laboral regular.

Con respecto a las estrategias se ha considerado elaborar una propuesta que permitan a los directivos implementar acciones correctivas, especialmente en las cuatro dimensiones que obtuvieron un nivel regular de clima laboral. En este caso a las dimensiones de recompensas, comunicación, cooperación / Apoyo y conflictos; mismas que están enfocadas a elevar la motivación de los colaboradores, implementar nuevas técnicas de comunicación internas para transmitir la información, realizar gestiones para procurar arreglos satisfactorios por causa de un conflicto y también fomentar el espíritu de trabajo en equipo. Para lo cual se consideró un presupuesto total de \$6404.

Por último, al analizar las distintas interpretaciones se evidencia que todos los autores llegan a la conclusión sobre el concepto de clima laboral como los rasgos psicológicos y físicos que los colaboradores tienen sobre la organización y de su forma de comportarse dentro de la misma, lo cual se traduce en garantizar satisfacción y productividad.

\section{Referencias}

Cardona, D., \& Zambrano, R. (2014). Revisión de instrumentos de evaluación de clima organizacional. Estudios Gerenciales, 30, 184-189. Obtenido de https://www.sciencedirect.com/science/article/pii/S012359231400117X

Epín, M., Zula , J., Espín, E., \& Carrión, L. (2015). Gestión del Talento Humano Orientado en el Alto Desempeño de los Servidores Públicos . Eumed.net. Obtenido de http://www.eumed.net/rev/cccss/2015/03/talento-humano.html

GADPCH. (2016). (G. A. Chimborazo, Productor) Recuperado el 05 de 2018, de http://www.chimborazo.gob.ec/chimborazo/?p=96

Jiménez, H., \& Mosquera , A. (2017). Clima Organizacional y su Incidencia en el Desempeño Laboral de los Departamentos Financieros en Entidades Públicas. Quito, Pichincha. Obtenido de http://repositorio.upacifico.edu.ec/bitstream/40000/307/1/TNE_UPAC_17699.pd

Juárez, S. (2012). Clima Laboral y Satisfacción Laboral. Redalyc, 50(3), 307-

314. Obtenido de http://www.redalyc.org/articulo.oa?id=457745495014

Litwin, G., \& Stringer , H. (1968). Motivation and Organiational Climate. Boston: Harvard Business School.

Montoya, C., \& Boyero, M. (2015). El Recurso Humano como Elemento Fundamental para la Gestión de Calidad y la Competitvidad Organizacional. Redalyc, 20(2), 1-20. Obtenido de

http://www.redalyc.org/pdf/3579/357947335001.pdf 


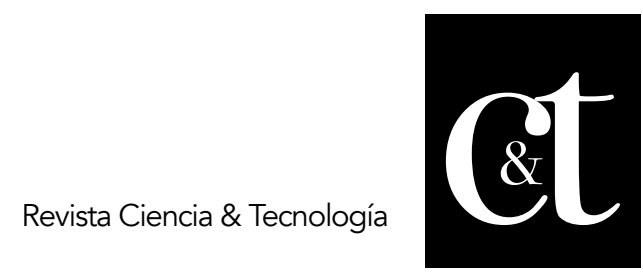

No. 29, 31 de enero de 2021

ISSN impreso: 1390 - 6321

ISSN online: 2661 - 6734

Montoya, D. (2016). Relación entre el Clima Organizacional y la Evaluación del Desempeño del Personal en una Empresa de Servicios Turísticos: Caso PTS Perú 2015. Lima, Perú. Obtenido de file://C:/Users/ruddy/Downloads/MONTOYA_MEZA_DANIEL_RELACI\%C3\%9 $3 N . p d f$

Olaz, Á. (2013). El Clima Laboral en Cuestión, Revisión Bibliográfica - Descriptiva y Aproximación a un Modelo Explicativo Multivariable. Redalyc(56), 1-35. Obtenido de http://www.redalyc.org/articulo.oa?id=495950252002

Pecino, V., Mañas , M., Díaz , P., López, J., \& Llopis, J. (2015). Clima y satisfacción laboral en el contexxto universitario. Redalyc, 31(2), 658-666. Obtenido de http://www.redalyc.org/articulo.oa?id=16738685030 